\title{
Diagnosis and management of methicillin resistant Staphylococcus aureus infection
}

\author{
Georgia J Duckworth
}

\begin{abstract}
Methicillin resistance in Staphylococcus aureus
Staphylococcus aureus is renowned for countering almost any antibiotic challenge. Its history since the advent of penicillin is one of increasing resistance to antibiotics. Methicillin was the first semisynthetic penicillin resistant to penicillinase to be derived from the penicillin nucleus and was a major advance in the treatment of staphylococcal infections. Because of its toxicity and ineffectiveness if given orally it was gradually superseded by newer penicillinase resistant penicillins such as flucloxacillin. However, methicillin is still used for susceptibility testing of staphylococci in the laboratory and so methicillin resistance is an indicator of flucloxacillin resistance. Staphylococcal resistance to methicillin is not due to the destruction of the antibiotic by a bacterial enzyme such as $\beta$-lactamase but is related to decreased affinity of the organism's penicillin binding proteins for methicillin and also the synthesis of an extra penicillin binding protein with very low affinity for $\beta$-lactam antibiotics.
\end{abstract}

Methicillin resistance in staphylococci was reported in 1961, soon after the introduction of methicillin, and naturally occurring strains may have been around before the advent of methicillin. ${ }^{1-3}$ There was a gradual increase in methicillin resistance in England throughout the 1960s, but during the 1970s attention was focused on the Gram negative bacteria, with some complacency about Gram positive bacteria.

Various epidemic strains of methicillin resistant $S$ aureus (EMRSA) appeared in the 1980s. ${ }^{4}$ The first of these, EMRSA-1, bore many resemblances to a strain that caused large outbreaks in hospitals in eastern Australia in the late 1970 s. $^{5}$ It probably first appeared in England in 1980-1 and went on to cause major endemic and epidemic problems. These were centred in hospitals in the North East Thames region of London, but also spread widely to hospitals in other parts of London and outside London. ${ }^{6-8}$ Since then

Department of Medical Microbiology, London Hospital Medical College, London E1 2AD

Georgia J Duckworth, senior lecturer

BMF 1993;307:1049-52
Box 1-Methicillin resistance in

Staphylococcus aureus

- Methicillin resistance indicates flucloxacillin resistance

- There are many different strains of MRSA

- MRSA strains are usually resistant to many other antimicrobials

- Methicillin resistance is not a marker for virulence or spread

- Some MRSA have epidemic potential

- MRSA may possess all the Staphylococcus aureus virulence factors

- MRSA may cause severe infections or merely colonisation

Summary points
- Control of methicillin resistant Staphylococcus
aureus (MRSA) is difficult and costly
- Management of infections has two aspects:
management of the individual patient and
management of an endemic or epidemic prob-
lem with MRSA in a hospital
- Control of epidemics relies essentially on
good hygienic practices and hospital policies to
restrict the use of antibiotics
The costs of ignoring an outbreak are higher
than those associated with its control, particu-
larly when the costs of potential legal action are
taken into account

many other epidemic strains have been identified. For instance, a new strain, termed EMRSA-16, was recognised in an outbreak in the orthopaedic and geriatric wards of a hospital in Northamptonshire in 1991. This went on to become established in two district general hospitals and a London teaching hospital in 1992, and isolates were received from 13 other hospitals in England and Wales. ${ }^{9}$

These later strains may have evolved from the earlier ones, but they are distinctly different. In particular, many of the resistance determinants in the newer strains are chromosomal rather than plasmid-borne, which may stabilise them. It is now clear that genetic information may be transferred by a fairly promiscuous process between ubiquitous, relatively nonpathogenic organisms and pathogenic organisms sharing overlapping habitats (for example, coagulase negative staphylococci, $S$ aureus, Streptococcus pyogenes, Streptococcus agalactiae, and Enterococcus faecalis)..$^{10-12}$ This development has worrying implications. Add the selective pressures of topical and systemic antibiotics, compromised patients, invasive procedures, and the hospital environment and it is easy to imagine how outbreaks with multiresistant strains of $S$ aureus may arise and persist.

\section{Virulence and epidemic potential}

Arguments rage about the virulence and epidemic potential of strains of methicillin resistant $S$ aureus. These bacteria are largely hospital pathogens and it is often argued that they behave as opportunistic pathogens. However, they are a heterogeneous group of organisms and as such do not all behave in the same way (box 1). It is clear that methicillin resistance itself 
is not a marker of epidemic potential or virulence, but it is still unclear which factors are linked to these aspects of behaviour. Virulence factors in these staphylococci tend to be those of the more common methicillin sensitive $S$ aureus (MSSA). In general, the resistant staphylococci are as capable as the sensitive staphylococci at producing the impressive array of factors implicated in virulence and pathogenicity such as coagulase, DNAse, haemolysins, protein $\mathrm{A}$, and other extracellular toxins.

Methicillin resistant $S$ aureus may cause severe and life threatening infections. Studies have case matched patients with infections due to methicillin sensitive and methicillin resistant bacteria and found no significant differences in the two groups, and in Dublin methicillin resistant $S$ aureus bacteraemias had a high case fatality rate. ${ }^{13}$ In an American outbreak $30 \%$ of all bacteraemias in a three year period were caused by methicillin resistant $S$ aureus, which was a direct cause of death in $44 \%$ of these patients. ${ }^{14}$ This study also reported a high rate of infection, 260 of 286 affected patients being infected and not simply colonised. Other places in England and abroad have had similar experiences. In the Royal London Hospital's EMRSA-1 outbreak severe infections (septicaemia, endocarditis, meningitis) were notable in the early days, before full containment measures were instituted. ${ }^{6}$ In this outbreak there were also two instances where EMRSA-1 was reponsible for a toxic shock-like illness; this was probably associated with enterotoxin production as toxic shock syndrome toxin-1 was not isolated from these strains. ${ }^{15}$

The transmission and acquisition of methicillin resistant $S$ aureus is a multifactorial process depending not only on organism factors but also on host factors such as abnormal or damaged skin, length of hospital stay, previous antibiotic treatment, and probably the use of topical antimicrobial agents. There also seems to be genetic predisposition to carriage of $S$ aureus, or lack of it. Not surprisingly, the hospital areas mainly affected are the intensive care, burns, dermatology, and surgical units.

\section{Diagnosis}

It is not possible to diagnose methicillin resistant $S$ aureus infection from the patient's clinical condition as there is no characteristic clinical picture that differentiates this from other staphylococcal infections. Diagnosis of infection or colonisation will depend on the submission of appropriate specimens to the microbiology laboratory. When infection is suspected these should be from the site or sites of infection, whereas screening for colonisation (box 2) should include samples from the nose, throat, wounds, other skin lesions, insertion sites of intravascular lines, tracheostomies, catheter urine samples, perineum, and sputum. The usual strictures apply: samples should always be fresh; actual pus is better than a wound swab; and swabs in transport medium are better than dry swabs. Normal sampling methods to detect colonisation are relatively insensitive, so several sets of clear specimens are required before a patient can be declared free of

\section{Box 2-Sampling sites to detect colonisation}

- Nose

- Throat

- Perineum

- Sites of abnormal or damaged skin

- Wounds

- Insertion sites of intravascular lines

- Catheter urine samples

- Sputum if expectorating
Box 3-Procedure for colonised or infected patients

- Discharge patients with instructions for clearing MRSA colonisation if their condition allows

- If discharge is not possible, admit them to an isolation room or ward

- Submit further cultures to assess extent of colonisation and dispersal

- Assess whether MRSA strain has previously exhibited epidemic potential

- Mark the patient's records for future recognition

- Treat infection with the appropriate antimicrobial agents

- Treat colonisation with antiseptics and topical agents

- Assess the vulnerability of the other patients on the ward

- Consider screening other patients and staff in areas where the patient has been, to detect other affected people or the source

- Reinforce hygienic precautions and infection control measures, including restrictions on the use of antibiotics

- Decide whether the ward should be closed to admissions and thoroughly cleaned

methicillin resistant $S$ aureus. During a screening operation, sensitivity of detection can be improved by placing the swabs directly into a salt enrichment broth on the ward. However, this delays the result by a further day compared with a direct plate culture on blood or nutrient agar.

The detection of methicillin resistance poses problems for the microbiologist as its expression is affected by many physical and chemical factors, such as light, temperature, osmolality, and $\mathrm{pH} .{ }^{16-19}$ This may result in a population seeming methicillin sensitive unless culture conditions are manipulated to bring out methicillin resistance. This is commonly done either by incubating the strain at $30^{\circ} \mathrm{C}$ or by adding extra salt to the medium. Further difficulties arise in typing the staphylococcus, as many strains are untypable by the standard phages.

\section{Managing patients with MRSA}

ISOLATION

Containment measures should be instituted when evidence of methicillin resistant $S$ aureus has been obtained from a patient as it is not possible to determine the behaviour of the particular strain from laboratory information. The patient should be admitted to an isolation room or unit. The need for scrupulous attention to hand washing by staff must be reinforced, as most staphylococci are spread by the hands. Transmission through the air may occur and is most likely with heavily colonised people, termed "dispersers" or "cloud babies." Skin conditions such as eczema predispose to widespread dispersal of staphylococci on skin scales through the air. It is virtually impossible to clear methicillin resistant $S$ aureus from such people, which has serious consequences if the affected person is a health care worker. Sideroom isolation may fail where air dispersal is a factor or where hand hygiene of staff is unsatisfactory. In these circumstances isolation in a dedicated isolation ward with staff well versed in infection control procedures may be necessary (boxes 3 and 4).

\section{TREATMENTS}

The treatment of patients affected with methicillin resistant $S$ aureus falls into two categories: treatment of colonisation and treatment of infection. The ablation of widespread colonisation (boxes 5 and 6) presents a problem as none of the currently available antiseptics 
(hexachlorophane, triclosan, chlorhexidine, povidoneiodine) is fully effective, although they are used in hair and body washing for want of something better. The treatment of limited carriage, particularly nasal carriage, has been revolutionised by mupirocin, ${ }^{20}$ but reports of reduced susceptibility to this agent should alert us to the danger of excessive or inappropriate use. ${ }^{21}$ In some instances carriage may not be ablated by topical agents or by systemic treatment of infection, and recourse to rifampicin may be necessary, possibly in combination with ciprofloxacin.

Strains of $S$ aureus vary in their degree of methicillin resistance and in their pattern of resistance to other antimicrobial agents. Most are resistant to penicillin and susceptible to fusidic acid, rifampicin, teicoplanin, and vancomycin, but the rest of their antibiogram varies widely. EMRSA-1 is characteristically resistant to penicillin, tetracycline, erythromycin, methicillin, and clindamycin; variably resistant to gentamicin and chloramphenicol; usually sensitive to neomycin, fusidic acid, ciprofloxacin, and rifampicin in patients not receiving these drugs; and consistently sensitive only to vancomycin and teicoplanin.

\section{Box 4-Prevention of spread of MRSA}

- Isolation of affected patient; removal and treatment of affected staff

- Thorough hand washing before and after contact with affected patients or their immediate environment

- Gloves for handling contaminated dressings or linen

- Masks for procedures which generate staphylococcal aerosols (chest physiotherapy, sputum suction, etc)

- Gowns or plastic aprons for close contact with patients or their immediate environment

- Disposal of waste and linen according to policy for "infected" materials and linen

- Terminal room disinfection with a phenolic disinfectant, with special attention to horizontal surfaces and areas that collect dust

- Defined procedures for patient's visits to specialist departments, surgery, ambulance transportation, transfer to another hospital, etc: MRSA patients should normally be dealt with at the end of the working session

- Limit use of agency staff in affected areas

There are formidable problems in the treatment of methicillin resistant $S$ aureus infections (box 7), given the scarcity of available agents. The mainstay of treatment is vancomycin, although experience with a newer glycopeptide, teicoplanin, is growing. ${ }^{22} 23$ Teicoplanin may replace vancomycin as it is easier to administer and has a longer half life. It may be less toxic than vancomycin, although reports of vancomycin toxicity have been exaggerated and probably relate to the earlier, dirtier product, the "Mississippi mud" of the 1950s.

Vancomycin is cheaper but requires careful monitoring and slow infusion. Tolerance may occur, requiring combination therapy, for example with rifampicin. Rifampicin is an excellent antistaphylo-

\section{Box 5-Treatment of colonisation}

- Skin and hair: antiseptic detergent for washing to decrease the staphylococcal load

- Nose and sites of limited carriage: mupirocin Chlorhexidine based creams are less effective but may be required if MRSA is resistant to mupirocin

- Antibiotics such as rifampicin and ciprofloxacin may occasionally be required to eradicate colonisation

\section{Box 6-Clearance of MRSA}

- Sample nose, perineum, skin lesions, and other previously positive sites weekly

- The patient is deemed clear of MRSA if results of three complete sets of screening tests are negative

- Relapses may occur if the patient receives antibiotics later

coccal agent, but resistance arises readily if it is used alone. ${ }^{2426}$ There are also concerns that its widespread use may jeopardise its value in the treatment of tuberculosis. Some of the newer quinolones such as ciprofloxacin have antistaphylococcal activity, but resistance also arises and treatment failure despite continued susceptibility of the organism to ciprofloxacin has been reported. ${ }^{27} 28$ These agents may have a role in the treatment of non-life threatening infections.

\section{Box 7-Treatment of infection}

- Severe or life threatening infection: vancomycin or teicoplanin. Combination therapy may be required-for example, with rifampicin

- Non-life threatening infection: appropriate treatment according to the antibiotic susceptibility of the strain of $S$ aureus

\section{Management of endemic or epidemic MRSA}

Control rests on good hygienic practices and hospital policies to restrict the use of antibiotics. Hospital surveillance and an awareness of other local units and hospitals similarly affected are crucial. There needs to be prompt recognition of cases and impending outbreaks. The necessary containment measures will vary according to the epidemic potential of the methicillin resistant $S$ aureus, but this is not usually known in advance. Experience with some strains of methicillin resistant $S$ aureus, including EMRSA-1, shows that isolation or cohort wards may be necessary. ${ }^{6}$ Surveillance of high risk admissions, screening of wards with affected patients, the tagging of affected patients' records, and the restriction of staff and patient movement within and between hospitals are also important aspects of control and are detailed in the revised guidelines for the control of epidemic methicillin resistant $S$ aureus. ${ }^{29}$ In some instances such as an endemic problem in a less critical area of the hospital, eradication of the methicillin resistant strain may prove impossible, requiring an acceptance of living with it. Control here may mean limiting spread outside that area.

\section{The future}

As yet little is known about the factors that make a particular strain of methicillin resistant $S$ aureus virulent or capable of spread. The rapid identification of epidemic strains and the factors responsible for spread and colonisation would permit a more targeted approach and save on containment measures. Other areas warranting further research include the development of new topical and systemic agents, more rapid and sensitive methods of detecting colonisation, and improved typing methods.

An outbreak of methicillin resistant $S$ aureus is costly business (box 8). It is generally agreed that the costs of ignoring EMRSA are higher than those associated with its control, particularly when the costs of potential legal action are taken into account. The average cost of treating a bacteraemia with methicillin 


\section{Box 8-Costs of an MRSA outbreak}

- Cost to patients: pain and discomfort, loss of time and earnings, inconvenience, excess morbidity, and mortality

- Cost to the hospital: delayed discharge and attendant costs, drugs and antiseptics, containment measures, infection control procedures, litigation, contracts imperilled, exclusion of affected staff

resistant $S$ aureus is about seven times that of treating a bacteraemia with methicillin sensitive $S$ aureus. ${ }^{30}$ Containment measures use increasingly scarce resources. A 1987 study in the North East Thames region put the recurrent annual financial cost then of containing outbreaks at around $£ 250000$ in the worst affected hospitals. In addition, there were capital costs associated with the establishment of an isolation ward. Another cost that must not be neglected is that of litigation by infected patients, a growing hazard of outbreaks in hospitals. Hospitals then need to show that well documented and effective control measures are in place. Furthermore, infection control now features as one of the main quality indicators in contracts between purchaser and provider agencies.

1 Jevons MP. “Celbenin" resistant staphylococci. BMf 1961;i:124-5.

Cetin ET, Ang O. Staphylococci resistant to methicillin ("Celbenin"). BM 1962;ii:51-2.

3 Borowski J, Kamienska K, Rutecka I. Methicillin resistant staphylococci. $B M 7$ 1964;: 983 .

4 Combined Working Party of the Hospital Infection Society and British Society for Antimicrobial Chemotherapy. Guidelines for the control of epidemic methicillin-resistant Staphylococcus aureus. I Hosp Infect 1986;7:193-201.

5 Townsend DE, Ashdown N, Bradley JM, Pearman JW, Grubb WB. "Australian" methicillin-resistant Staphylococcus aureus in a London hospital? Med I Aust 1984;i: 339-40.

6 Duckworth GJ, Lothian JLE, Williams ID. Methicillin-resistant Staphylococcus aureus: report of an outbreak in a London teaching hospital. $f$ Hosp Infect 1988;11:1-15.

7 Bradley JM, Noone P, Townsend DE, Grubb WB. Methicillin-resistan Staphylococcus aureus in a London hospital. Lancet 1985;i:1493-5.

8 Cooke EM, Marples RR. Methicillin resistant Staphylococcus aureus. BM 1987;294:371.
9 EMRSA-16: a new epidemic strain of Staphylococcus aureus. Communicable Disease Report 1993;3:25.

10 Schaberg DR, Clewell DB, Glatzer L. Conjugative transfer of R-plasmids from Streptococcus faecalis to Staphylococcus aureus. Antimicrob Agents Chemother 1982;22:204-7.

11 Soedirman EN, Rost JA, van Leeuwen WJ, van Embden JDA. Transferability of macrolide, lincomycin and streptogramin resistances between group A, B and $D$ streptococci, Streptococcus pneumoniae and Staphylococcus aureus. f Bacteriol 1980;142:407-13.

12 Collatz E, Carlier C, Courvalin P. The chromosomal 3',5"-paminoglycoside phosphotransferase in Streptococcus pneumoniae is closely related to its plasmid-coded homologs in Streptococcus faecalis and Staphylococcus aureus. F Bacteriol 1983;156:1373-7.

13 Hone R, Cafferkey M, Keane CT, Harte-Barty M, Moorhouse E, Carroll R, et al. Bacteraemia in Dublin due to gentamicin-resistant Staphylococcus aureus. $\mathcal{F}$ Hosp Infect 1981;2:119-26.

14 Myers JP, Linnemann CC. Bacteraemia due to methicillin-resistant Staphylococcus aureus. FInfect Dis 1982;145:532-6.

15 Duckworth GJ, Oppenheim BA. Enterotoxin production in epidemic methicillin-resistant Staphylococcus aureus. Lancet 1986;i:565-6. methicillin-resistant Staphylococcus aureus. Lancet 1986;i:565-6.
Knox R. “Celbenin" resistant staphylococci. BMF 1961;i:126.

17 Benner EJ, Morthland V. Methicillin-resistant Staphylococcus aureus. Antimicrobial susceptibility. N Engl f Med 1967;277:678-80.

18 Annear DI. The effect of temperature on resistance of Staphylococcu aureus to methicillin and some other antibiotics. Med $\mathcal{F}$ Aust 1968;i:444-6.

19 Sabath LD. Chemical and physical factors influencing methicillin resistance of Staphylococcus aureus and Staphylococcus epidermidis. I Antimicrob Chemother 1977;3(suppl C):47-51.

20 Hill RLR, Duckworth GJ, Casewell MW. Elimination of nasal carriage of methicillin-resistant Staphylococcus aureus with mupirocin during a hospital outbreak. I Antimicrob Chemother 1988;22:377-84.

21 Baird D, Coia J. Mupirocin-resistant Staphylococcus aureus. Lancet 1987;ii: 387-8.

22 Woodley DW, Hall WH. The treatment of severe staphylococcal infections with vancomycin. Ann Intern Med 1961;55:235-49.

23 Cafferkey MT, Hone R, Keane CT. Severe staphylococcal infections treated with vancomycin. $₹$ Antimicrob Chemother 1982;9:69-74.

24 Mandell GL, Vest TK. Killing of intraleukocytic Staphylococcus aureus by rifampin: in-vitro and in-vivo studies. $\mathcal{F}$ Infect Dis 1972;125:486-90.

25 Jensen K. Methicillin-resistant staphylococci. Lancet 1968;ii:1078.

26 Gruneberg RN, Emmerson AM. Failure to prevent emergence of bacteria resistance by combination of two antibiotics: fusidic acid and rifampicin I Antimicrob Chemother 1980;6:562-4.

27 Foster JK, Lentino JR, Strodtman R, DiVincenzo C. Comparison of in vitro activity of quinoline antibiotics and vancomycin against gentamicin- and methicillin-resistant Staphylococcus aureus by time-kill kinetic studies. Antimicrob Agents Chemother 1986;30:823-7.

28 Righter J. Ciprofloxacin treatment of Staphylococcus aureus infections. IAntimicrob Chemother 1987;20:595-7.

29 Duckworth G, Ayliffe GAJ, Brumfit W, Casewell MW, Cooke EM, Cookson BD, et al. Report of a combined working party of the Hospital Infection Society and British Society for Antimicrobial Chemotherapy. Revised Society and British Society for Antimicrobial Chemotherapy. Revised
guidelines for the control of epidemic methicillin-resistant Staphylococcus aureus. I Hosp Infect 1990;16:351-77.

30 Cheng AF, French G. Methicillin-resistant Staphylococcus aureu bacteraemia in Hong Kong. $\mathcal{F}$ Hosp Infect 1988; 12:91-101.

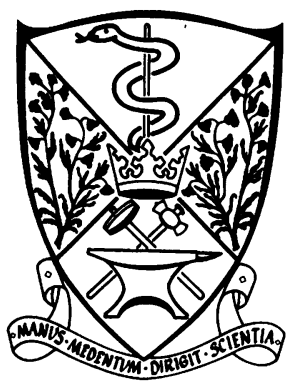

Royal Postgraduate Medical School, London W12 0NN

Case presented by: C P Clifford, cardiology registrar

G J Davies, consultant cardiologist

Chairman:

Discussion Group:

J Scott, professor of medicine

$S$ Shaunak, consultant in infectious disease

J Savill, Wellcome Trust senior research fellow in clinical science

J B Schofield, histopathology senior registrar

Series edited by:

Dr Moira Whyte.

BMF 1993;307:1052-4

\section{Grand Rounds: Hammersmith Hospital}

\section{Tuberculous pericarditis with rapid progression to constriction}

\section{Prompt diagnosis and treatment are needed}

Pericarditis is the main diagnosis in 1 in 1000 patients admitted to hospital, and $4 \%$ of cases are caused by tuberculosis. ${ }^{1}$ Tuberculosis is responsible for up to $7 \%$ of cases of pericardial tamponade and a similar percentage of cases of tamponade in which the patient requires pericardiectomy because pericardial constriction develops. ${ }^{2}$ We report a case of tuberculous pericarditis in which pericardial constriction developed rapidly despite anti-tuberculous drugs.

\section{Case history}

A 28 year old Sri Lankan man was transferred to our hospital for investigation and treatment. He had been admitted to another hospital three days previously with a four week history of malaise, loss of appetite, weight loss $(5 \mathrm{~kg})$, reduced exercise tolerance, and rigors. $\mathrm{He}$ had recently arrived in England from Sri Lanka and had no significant medical history. $\mathrm{He}$ was heterosexual. On examination he had a temperature of $39^{\circ} \mathrm{C}$, his pulse was 100 beats/min with a palpable paradox, and his blood pressure was $120 / 70 \mathrm{~mm} \mathrm{Hg}$ with $20 \mathrm{~mm} \mathrm{Hg}$ of paradox. The jugular venous pressure was raised by $7 \mathrm{~cm}$ and Kussmaul's sign was present. A third heart sound was heard together with bibasal crepitations. He had hepatosplenomegaly but no ascites or peripheral oedema.

His haemoglobin concentration was $123 \mathrm{~g} / \mathrm{l}$, white blood cell count $12.4 \times 10^{3} / 1$, platelet count $370 \times 10^{9} / 1$, and the erythrocyte sedimentation rate $48 \mathrm{~mm}$ in the first hour. His biochemical profile was normal but his $C$ reactive protein concentration was $200 \mathrm{mg} / \mathrm{l}$. Three early morning urine samples yielded no growth. An electrocardiogram showed a sinus tachycardia, with a rate of 119 beats/minute, with $T$ wave inversion in the inferolateral leads. Chest radiography showed cardiomegaly (fig 1). He had good biventricular function, normal valvular structure and function, but a large pericardial effusion (fig 2). Pericardiocentesis yielded 1.5 litres of uniformly blood stained fluid. The fluid contained an exudate with a protein concentration of $540 \mathrm{~g} /$, glucose concentration of $1.9 \mathrm{mmol} / \mathrm{l}$ (serum glucose $5 \mathrm{mmol} / \mathrm{l}$ ), and numerous lymphocytes. A Mantoux test was performed and gave positive results at a dilution of 1 in 10000 . Computed tomography showed evidence of para-aortic and paracolic lymphadenopathy. On the basis of fever, lymphadenopathy, and the large pericardial effusion he was started on 